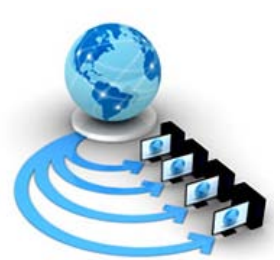

\title{
CONTENT-BASED IMAGE RETRIEVAL TECHNIQUES FOR MAMMOGRAPHIC IMAGES USING SOFT COMPUTING TECHNIQUES
}

\author{
Er. Sumit Chopra \\ Research Scholar \\ IKG Punjab Technical University \\ Kapurthala, India
}

\author{
Dr. V. K. Banga \\ Principal \\ Amritsar College of Engineering and Technology \\ Amritsar, India
}

\begin{abstract}
Content-based image Retrieval has been one of the lively research in the field of the computer science over the last decade or so. Many programs and tools have been used to formulate and execute queries based on the visual or audio content and to help browsing large multimedia repositories. But still a lot of work needs to be done in case of mammographic images. Breast cancer has been one of the causes of the increasing death of the European women. Early diagnosis of the breast cancer can save one's life while incorrect diagnosis can lead a patient to unwanted stress and treatment. In this paper, a survey of the techniques used for the various steps of Content-Based Image Retrieval of Mammograms is done. Section 1 gives the general introduction to Content-Based Image Retrieval for Mammographic Images. Section 2 details about the various techniques used for pre-processing of the Mammographic images. Section 3 provided a survey of the various algorithms used for segmentation of the pectoral muscles from the mammogram. Section 4 briefs about various feature extraction and selection methods for mammographic images. Section 5 survey the various algorithms for detection and classification of abnormalities in mammograms.
\end{abstract}

Keywords: Mammograms, BIRADS (Breast Image Reporting and Data System), Breast cancer, Support Vector Machines, Pectoral Muscle

\section{INTRODUCTION}

Breast cancer is one of the most common cancers among women. In the United Kingdom, breast cancer accounts for $30 \%$ of all female patients with cancer and approximately 1 in 9 may suffer from breast cancer sometimes during their life [1]. Although breast cancer is a fatal disease, patients still have high chances of survival if the malignancy is detected at an early stage. Unfortunately, a high percentage of breast cancer cases are overlooked by the radiologist during routine screening. While false negatives can cost lives, false positive can cause panic and lead to unnecessary treatment. It has been reported that only $15-34 \%$ of the patients subjected to biopsy are found to actually have malignancies. Medical frameworks have been developed to support the development and installation of a system for content-based image retrieval in medical applications [2].

For research work in mammographic images, mainly two databases namely Mammographic Image Analysis Society Digital Mammogram Database and Digital Database for Screening Mammography are used. The MIAS Database is composed of a set of 322 MLO Digitized Mammograms corresponding to left and right breast of 161 women. The films were extracted from the UK National Breast Screening Programme and digitized to 50-micron pixel edge with a JoyceLoebl scanner, a device with a linear response in the optical density range 0-3.2.Each pixel was described as an 8-bit word. The database also includes "ground truth" on the location of any abnormalities may be present [3]. There are two views of mammography known as MLO and CC view and the effect of two view mammography versus single view mammography at subsequent screens on breast cancer detection and financial consequences for particular settings are shown in [34].

The Digital Database for Screening Mammography (Keath, Bowyer, Kopans, Moore and Kegelmeyer , 2000) of the University of South Florida is a large database of digitized mammograms available online. The database is divided into 43 volumes, and each volume is divided in a number of studies.
The grouping factor is the study final diagnostics: volume with normal cases, volume with a case containing benign abnormalities, a volume containing cases with cancerous abnormalities. In total, there are 2620 cases, and each case corresponds to the MLO and CC views of both women breasts, along with some associated patient information(age, breast density, rating and keyword description for abnormalities) and image information (scanner, spatial resolution). Moreover, images containing suspicious area have associated "ground truth" information about the location and type of suspicious regions.

\section{PREPROCESSING OF MAMMOGRAPHIC IMAGES}

Before any processing can be done on mammographic images, the first step is preprocessing of the mammographic images. With the preprocessing or the enhancement techniques, the fine details are also clearly visible and it becomes easier to segment mass and other abnormalities in mammographic images. One of the techniques that can be used for contrast enhancement of mammographic images is adaptive fuzzy contrast enhancement method. The mammograms are normalized and fuzzified based on maximum entropy principle. The local contrast was improved by using both local and global features and then defuzzified to transform the enhanced mammogram back into spatial domain [5]. The standard histogram equalization usually results in excessive contrast enhancement because of lack of contrast enhancement. The modified Local contrast enhancement can be used to adjust the level of contrast enhancement, which in turn gives the resultant image a strong contrast and also brings local details for more relevant interpretation [6]. Many variants of histogram equalization such as Contrast limited adaptive histogram equalization techniques and two-stage adaptive histogram equalization can be used for enhancement of mammograms [7, 8]. The limitation of existing contrast enhancement and brightness preserving technique for enhancing the digital 
mammograms is that they limit the amplification of the contrast by clipping the histogram at a predefined clip limit. To overcome this, fuzzy logic can be used in conjunction with histogram-based clipping algorithm to automate the selection of the clip limit that is relevant to mammograms [11].

The structure tensor operator can be combined with the fuzzy logic for improved enhancement of possible Micro calcifications in digital mammograms [9]. Nonlinear polynomial filters can be used for the design of Non-Linear Unsharp Masking for the enhancement of mammographic images. The different variants of Non-Linear Polynomial Filters can be used for different operational modules like edge preserving and contrast enhancement [10].

Breast image preprocessing is needed to improve the quality of the image. An image selection process should be incorporated in to better target problematic images [12]. In some circumstances, various enhancement algorithms can be combined together to achieve better results for the segmentation of the breast region and visual interpretation, analysis, and classification of mammogram masses to assist the radiologist in making more accurate decisions [13].

\section{PECTORAL MUSCLE SEGMENTATION}

After preprocessing of the mammogram image, the next step in the processing of the mammographic images is the removal of pectoral muscles so that the region of interest can be extracted easily. Pectoral muscles have pixel intensities similar to that of breast tissues which could lead to awry results. As a result, a lot of effort needs to be put into the segmentation of pectoral muscles and finding its contour with breast edges. A comprehensive survey of techniques used for segmentation of pectoral muscles is given in [21]. Adaptive pyramids and Minimum spanning trees can be used in conjunction with active contours to segment pectoral muscles in screening mammograms [14].Mammograms can also be segmented using multidimensional scanning of the image using a window of a particular size. The border pixels are detected using intensity values and a maximum gradient of the window. The breast boundary is identified from the detected pixels filtered using an averaging filter [15].

Breast skin-air interface segmentation is a much more difficult task when performed on scanned mammograms than digital mammograms. Pectoral muscle segmentation can be used by a combination of contrast enhancement using adaptive histogram equalization and polynomial curvature estimation on a selected region of interest. This method makes segmentation of very low contrast pectoral muscle areas possible because of estimation used to segment areas which have lower contrast difference than detection threshold [16]. Pectoral muscle segmentation can be done by means of two anatomical features namely homogeneous texture and high-intensity deviation which gives the initial pectoral muscle edge. Then the Kalman filter is used to refine the ragged edge [17]. Pectoral muscles can be also segmented by means of global thresholding, followed by edge detection processes to identify the edges of full breast and connected component labeling to identify and remove the connected pixels outside the breast region $[18,19]$.

Breast boundary can be detected initially via thresholding followed by Active contour models without edges to search for the actual boundary. It is superseded by postprocessing technique to correct the overestimated boundary caused by artifacts by Canny edge detection. Subsequently, five edge features were identified to find the edge that has the highest probability of being initial pectoral contour and search for actual boundary via contour growing [20].

\section{FEATURE EXTRACTION AND SELECTION FOR MAMMOGRAPHIC IMAGES}

Various features can be used for mammographic images in order to classify the region as mass or non- masses. After the segmentation of the region of interest, the next step in mammographic images is to extract features from the region of interest. The mammogram images can be filtered using Gabour wavelets and directional features are extracted at different orientation and frequencies. Principal Component analysis can be used for reducing the dimensions of filtered and unfiltered high dimensional data [22]. Contourlet coefficients can be employed as a feature extractor to obtain the contourlet coefficients. The features can be selected using a genetic algorithm which results in more compact and discriminative feature set [23]. The discriminating breast tissue patterns can be obtained by variants of Local Ternary Pattern and Local Phase Quantization. It shows very good results for distinguishing benign from malignant tissues [24].

Feature selection is the process of selecting an optimum subset of features from the enormous potential features available in the given domain after the image segmentation. A survey of existing feature selection algorithms for classification and clustering can be found in [31]. In this, the comparison between different algorithm with a categorizing framework based on search strategies, evaluation criteria, and data mining tasks with unattempted combinations was provided. The general guidelines to select significant features include following considerations: Discrimination, Reliability, Independence, and Optimality. According to what features are selected, the feature space is divided into three subspaces: intensity feature, geometric features and texture features [25]. To find best feature set and a suitable neural architecture for micro calcification classification, a neuro genetic algorithm can be used in digital mammograms [26]. Genetic Algorithm and Particle Swarm Optimization can also be used for selecting the optimal subset for feature selection [27]. A connectionist online feature selection technique is used to identify the set of good features from the set of features which are computed at few randomly selected positive and negative pixels [28]. Discriminant Analysis has also proved to be a good method for selecting the best features to distinguish between normal and abnormal regions [29]. Other alternative for optimal feature selection is by using multi objective Genetic algorithm [30]. Principal Component Analysis can be one alternative to reduce the dimensionality of filtered and unfiltered high dimensional data [22].

After segmenting the Region of interest from mammographic images, usually textural, intensity and shape features are extracted and suitable features are selected using Correlation based feature selection algorithm for classifying the ROI's as masses or non-masses [31]. Branch and bound algorithm can also be used in conjunction with the genetic algorithm for feature selection which provides the best optimal features [32]. An illustrative example of how existing feature selection algorithms can be integrated into a meta algorithm that can take advantage of individual algorithms is shown in [33]. An added advantage of doing so is to help a user employ a suitable algorithm without knowing the details of each algorithm. 


\section{CLASSIFICATION AND DETECTION OF ABNORMALITIES IN MAMMOGRAMS USING SOFT COMPUTING TECHNIQUES}

A computer aided diagnosis algorithm identifying breast nodule malignancy using multiple ultra sonography features and artificial neural network classifier was developed in [35]. The features were extracted from sonographic images through digital image processing and an artificial neural network then distinguished malignant nodules based on those features and results were compared using Receiver Operating Characteristic curve. A new system was developed to detect malignant masses on mammograms by observing the behavior of iris filter at different scales. A backpropogation neural network classifier was trained to reduce the number of false positives [36].

A method for automatic detection of mammographic masses was developed based on segmentation of regions via thresholding at multiple levels and set of features were computed from the segmented regions. A region ranking system was used to identify the region most likely to produce abnormalities based upon the features computed [37]. A medical image retrieval system was developed by extracting texture features using Modified Discrete Cosine Transform. The hierarchical similarity measure was used to reduce the search space in large database which reduced the effect of background in the image and improved the performance of medical image retrieval [38]. A computer aided detection system was developed to detect micro calcifications using multi scale filter bank based on the concept of second order partial derivatives. Firstly, the region of interest are identified by a multiresolution based histogram technique which is decomposed into sub-bands, the low-frequency sub band is suppressed and then the high - frequency sub bands which contain only nodule like structures are reconstructed. This structure is determined by the eigen values of the Hessian matrix [39].

An automatic breast classification methodology for the classification of the breast tissue into fatty, glandular and dense tissue is developed. After preprocessing, the pectoral muscle was suppressed and statistical features such as mean, standard deviation, smoothness, third moment, uniformity and entropy was extracted from the suspicious region and fed into support vector machine in order to classify them as fatty, glandular and dense tissues [40]. A content based image retrieval system was designed to retrieve mammographies from large medical image databases based on breast density according to four categories defined by the American College of Radiology and is integrated to the database of the image retrieval in medical applications. Two dimensional principal component was used in breast density texture characterization, in order to effectively represent texture and allows for dimensionality reduction and support vector machine was used for retrieval process [41]. A novel framework designed to cover content based information retrieval framework, designed to cover several medical applications and allowing the possibility of retrieval of incomplete medical cases of several images together with semantic information was presented in [42]. The framework relied on committee of decision trees and decision support tools to process the information and was tested on two heterogeneous medical datasets namely DRD and DDSM.

A Content based image retrieval system was designed which employees a query example to search for similar images in the database. The framework was divided into online and offline feature extraction. To identify the lesion type the system will ask the user to categorize the lesion as mass or calcification which helps the system link and utilize the most appropriate feature set. The user provides relevance feedback in order to refine searches further by tuning the relevance feedback functions [43]. A CAD system for mammographic masses that uses mutual information based template matching scheme with intelligently selected templates was designed in [44]. A computational methodology to help detect masses by specialists was developed in [45]. Cellular neural network was used to segment the regions that contain masses. The shapes of the regions were analyzed by various shape descriptors and texture was analyzed through geostatic functions and support vector machine was used to classify the candidate region as mass or non-masses.

A Content based image retrieval system was developed for helping medical professionals to seek mass lesions that are pathologically similar to a given example. Shape and margin features were extracted from mass lesions and similarity between query image and database image was done by means of hierarchical arrangement of features and weighing distance measure [46]. CBIR can also be designed using support vector ensemble [47]. A particle swarm optimization rule based extractor can also be used for diagnosis [48]. An alternative approach for detection of breast abnormalities in digital mammogram can be with the help of Particle Swarm Optimized Wavelet neural Network [49] .

For heterogeneous medical retrieval system, a unified learning framework was developed based on Full range Autoregressive model with the Bayesian approach in [50]. A novel feature descriptor based on local bit plane decoded pattern for indexing and retrieval of biomedical images [51]. A very fast method for breast cancer detection and segmentation in mammograms was developed by means of simple image processing operations [52]. A system to obtain salient regions with improved Region of Interest using graph based visual saliency methods was developed in [53] and performance was measured by Receiver Operating Characteristic curve. A Radial Basis Function neural network for mammogram classification based on Grey level Cooccurrence matrix was developed in [54]. An alternative method of mammogram classification was designed using Law's Texture Energy measure as texture feature extraction [55].

\section{SYSTEM EVALUATION}

In any general retrieval domain, it is very difficult to compare any two retrieval systems. A single example result does not reveal a great deal about the real performance of the system and is not objective as the best possible query result can be chosen by the author. This problematic in retrieval system evaluation is described in [56]. The most common measures that are used are common to the domains of information retrieval or content based image retrieval such as precision and recall defined as follows:-

$$
\text { Pr ecision }=\frac{\text { Number of relevant images retrieved }}{\text { Number of items retrieved }}
$$

$$
\text { Recall }=\frac{\text { Number of relevant images retrieved }}{\text { Number of relevant images }}
$$




\section{CONCLUSION}

This paper provided survey of techniques used for various steps in diagnosis of breast cancer in mammogram images. The techniques for pectoral muscle segmentation which usually hinder the segmentation of region of interest from mammogram were surveyed. Then the various mammographic features that are used for representation of region as mass or non-mass were studied. Usually, the number of features is large in number, so for proper representation, techniques for dimensionality reduction of features were studied. Lastly, the various algorithms for detection and classification of abnormality in mammograms were analyzed and their pros and cons were discussed.

\section{REFERENCES}

[1] K. Beaver and G. Witham, "Information needs of the informal careers of women treated for breast cancer," Elsevier European Journal of Oncology Nursing, Vol. 11, No. 1, pp. 16-25, February 2007.

[2] M. O. Guld , C. Thies , B. Fischer , and T. M. Lehmann , "A generic concept for the implementation of medical image retrieval system,” Elsevier International Journal of Medical Informatics , Vol. 26, No. 2-3, pp. 252- 259, Feburary-March, 2007.

[3] J. Suckling , J. Parker , D. R. Dance , S. M. Astely, I. Hutt, C.R.M. Boggis, I. Ricketts, E. Stamatakis, N, Cerneas, S. L. Kok, P. Taylor, D. Betal, and J. Savage "The Mammographic Image Analysis Society digital mammogram database," Proc. Workshop on Digital Mammography, Vol. 3, No. 2, pp. 211221,1994.

[4] M. Keath, K. Bowyer , D. Kopans , R. Moore, and P.J. Kegelmeyer, "The Digital Database for screening Mammography,” Proc. International Workshop on Digital Mammography, Vol. 5, No. 2, pp. 212-218, 2000.

[5] H. D. Cheng, and H. Xu , "A noval fuzzy logic approach to mammogram contrast enhancement," Elsevier Information Sciences , Vol. 148, No. 1-4, pp. 167-184, 2002.

[6] S. Sundram , K. Ramar , N. Arumugam, and G.Prabin, "Histogram Modified Local Contrast Enhancement for mammogram images,” Applied Soft Computing , Vol. 11, No. 8, pp. 5809-5816, December 2011.

[7] I. K. Maitra , S. Nag, and S.K. Bandyopadhay "“Technique for preprocessing of digital mammograms," Computer Methods and Programs in Biomedicine , Vol. 107 , No. 2, pp. 175-188, August 2012.

[8] S. Anand and S. Gayathri, “Mammogram image enhancement by two stage adaptive histogram equalization,” Elsevier Optik International Journal for Light and Electronic Optics , Vol. 126, No. 21, pp. 3150- 3152 , November 2015.

[9] J. Jiang , B. Yao, and A . M. Wason “ Integration of fuzzy logic and structure tesnor towards mammogram contrast enhancement," Computerized Medical Imaging and Graphics , Vol. 29 , pp. 83-90, 2005

[10] V. Bhateja, M. Misra, and S. Urooj, "Human visual system based unsharp masking for enhancement of mammographic images,” Elsevier Journal of Computational Science , Vol. 21, pp. 387-393, July 2017.

[11] S. Jenifer, S. Parasuraman, and A. Kadirvelu, “ Contrast enhancement and brightness preserving of digital mammograms using fuzzy clipped contrast limited adaptive histogram equalization algorithm," Applied soft computing, Vol. 42, pp. 167-177, 2016.

[12] He W. , Hogg P. , Juette A. , Denton E. R. E. , Zwiggelaar R. “ Breast image preprocessing for mammographic tissue segmentation," Computers in Biology and Medicine , Vol. 67, No. C, pp. 61-73, 2015.

[13] N. A. Najdawi, M. Biltawi, and S. Tedmori, "Mammogram image visual enhancement, mass segmentation and classification,” Elsevier Applied Soft Computing”, Vol. 35, No. C, pp. 175-185, October 2015.

[14] F. Ma , M. Bajger , J. P. Slavotinek, and M. J. Bottema, "Two graph theory based methods for identifying the pectoral muscles in mammograms," Elsevier Pattern Recognition , Vol. 40 , No. 9 , pp. 2592- 2602 , September 2007.

[15] P. Kus and I. Karagoz , "Fully automated gradient based breast boundary detection for digitized X - ray mammograms," Computers in Biology and Medicine , Vol. 42 , No. 1 , pp. 7582, January 2012.

[16] M. Mustra and M. Grgic “ Robust automatic breast and pectoral muscle segmentation from scanned mammograms, " Elsevier Signal Processing , Vol. 93 , No. 10 , pp. 2817-2827 , October 2013.

[17] Y. Li, H. Chen, Y. Yang, and N. Yang “ Pectoral muscle segmentation in mammograms based on homogeneous texture and intensity deviation, ” Vol. 46 , No. 3, pp. 681-691 , March 2013.

[18] S. Sreedevi and E. Sherly, " A Novel Approach for Removal of pectoral muscles in Digital Mammogram,” Proc. International Conf. on International Conference on Information and Communication Technologies , Vol. 46 , No. , pp. 1724-1731 ,2014.

[19] P.S.Vikhe, and V. R. Thool, "Intensity based Automatic Boundary Identification of PectoralMuscles in Mammograms," Proc. 7th International Conference on Communication, computing and Virtualization, Vol. 79, Issue , pp. 262-269, 2016

[20] A. Rampun , P. J. Morrow, B. W. Scotney, and J.Winder , “ Fully automated breast boundary and pectoral muscle segmentation in mammograms,”, Elsevier Artifical Intelligence in Medicine , Vol. 79, pp. 28-41, 2017.

[21] K. Ganesan , U. R. Acharaya, K. C. Chua, L. C. Min, and K. T. Abraham " Pectoral muscle segmentation: A review," Computer Methods and Programs in Biomedicine , Vol. 110 , No. 1, pp. 48-57, April 2013.

[22] I. Buciu and A. Gascad " Directional features for automatic tumor classification of mammogram images,” , Biomedical Signal Processing and Control, Vol. 6 , No. 4, pp. 370-378, October 2011.

[23] F. Moayedi , Z. Azimifar, R. Boostani, and S. Katebi “ Contourlet- based mammography mass classification using the SVM family,”Elsevier Computes in Biology and Medicine, Vol. 40 , No. 4, pp. 373-380, 2010.

[24] L. Nanni, S. Brahnam , and A. Lumini, “ A very high performing system to discriminate tissues in mammograms as malignant and beingn, " Elsevier Expert System With Applications, Vol. 39 , No. 2, pp. 1968-1971, 2012.

[25] H. D. Cheng , X. J. Shi, R. Min, L. M. Hu , X. P. Cai, and H. N. Du , "Approaches for automatic detection and classification of masses in mammograms,"Elsevier Pattern Recognition, Vol. 39 , No. 4, pp. 646-668, April 2006.

[26] B. Verma and P. Zhang, “ A novel - genetic algorithm to find the most significant combination of features in digital mammograms,” Applied Soft Computing , Vol. 7, No. 2, pp. 612-625, March, 2007.

[27] K. Geetha, K. Thanushkodi , and A. K. Kumar,"New particle Swarm Optimization For Feature Selection and Classification of Microcalcifications in Mammograms,"Proc. International Conference on Signal Processing, Communication and Networking, pp. 458-463, 2008.

[28] M. K. Pal, B. Bhowmick, S. K. Patel , S. Pal, and J. Das, “A multistage neural network aided system for detection of 
microcalcifications in digitized mammograms”, Neurocomputing, Vol. 71, No. 13, pp. 2625-2634, August 2008.

[29] M. Sameti , R. K. Ward, J. K. Parks , and B. Palcic "Image Feature Extraction in the Last Screening Mammograms Prior to Detection of Breast Cancer," IEEE Journal of Selected Topics in Signal Processing , Vol. 3, No. 1, pp. 46-52, Febuary 2009.

[30] M. Suganthi and M. Madheswaran, "An improved medical Decision Support System to Identifiy the Breast Cancer Using Mammogram,” Journal of Medical System, Vol. 36, No. 1, pp. 79-91, Feburary 2012.

[31] W. Han, J. Dong, Y. Guo, M. Zhang, and J. Wang, "Identification of masses in digital mammogram using optimal set of features," Proc. IEEE $10^{\text {th }}$ International Conference on Trust, Security and Privacy in Computing and Communication , pp. 1763-1768, 2011.

[32] A. K. Mohanty, M. R. Senapati, and S. K. Lenka, “A novel image minning technique for classification of mammograms using hybrid feature selection," Neurocomputing and Applications , Vol. 22, No. 6, pp. 1151-1161, May 2013.

[33] H. Liu and L. Yu, "Towards Integrating Feature Selection Algorithms for Classification and Clustering," Proc. IEEE Transactions on Knowledge and Data Engineering , Vol. 17 , No. 4, pp. 491- 502 , April 2005.

[34] V. V. B. Smallenburg , L. E. M. Duijm , G. J. D. Heetan, J. H. Groenewoud, F. H. Jansen, J. Fracheboud, M. L. Plaisier , H. J. V. D. Nagtegaal, and J. M. Broeders, "Two - view versus single - view mammography at subsequent screening in a region of the Dutch breast screening programme, " European Journal of Radiology , Vol. 81 , No. 9 , pp. 2189- 2194, September 2012.

[35] S. Joo , W. K. Moon, and H. C. Kim "Computer- aided Diagnosis of Solid Breast Nodules on Ultrasound with Digital Image Processing and Artificial Neural Network," Proc. International Conference IEEE Engineering in Medicine and Biology Society , pp. 1397-1400 , San Francisco , CA,September 2004.

[36] C. Varela , P. G. Tahoces , A. J. Mendez, M. Souto, and J. J. Vidal, " Computerized detection of breast masses in digital mammograms, ” Elsevier Journal of Computers in Biology and Medicine, Vol. 37 , No. 2 , pp. 214- 226 , February 2007.

[37] A. R. Dominguez and A. K. Nandi , " Detection of masses in mammograms via statistically based enhancement, multilevelthresholding segmentation and region selection," Elsevier Journal of Computerized Medical Imaging and Graphics , Vol. 32 , No. 4, pp. 304-315, 2008

[38] K. Rajakumar and S. Muttan, “ Medical Image retrieval using modified DCT, ” Elsevier Procedia Computer Science, Vol. 2 , No. , pp. 298-302, 2010.

[39] T. Balakumaran ,I. L. A. Vennila, and C. G. Shankar , "Micro calcificationDetection in Digital Mammograms using Novel Filter bank," Proc. International Conference and Exhibition on Biometrics Technology , Vol. 2 , Issue , pp. 272282, 2010

[40] T. S. Subashini, V. Ramalingam, and S. Palanivel "Automated assessment of breast tissue density in digital mammograms," Elsevier Computer Vision and Image Understanding, Vol. 114, No. 1, pp. 33-43, January 2010

[41] J. E. E. D. Oliveira, A. M. C. Machado, G. C. Chavez, A. P. B. Lopes, T. M. Deserno, and A. D. A. Araujo,

MammoSys : A content-based image retrieval system using breast density patterns," Elsevier Computer Methods and Programs in Biomedicine , Vol. 99 , No. 3, pp. 289-297, March 2010.

[42] G. Quellec, M. Lamard , L. Bekri, and G. Cazuguel, "Medical Case Retrieval From a Committee of Decision Trees” Proc. IEEE Transactions on Information Technology in Biomedicine , Vol. 14, No. 5, pp. 1227- 1235, Sept. 2010.
[43] C. H. Wei , Y. Li, and P. J. Huang, “ Mammogram retrieval through machine learning within BI-RADS standards” Elsevier Journal of Biomedical Informatics, Vol. 44, No. 4, pp. 607-614 , August 2011.

[44] M. A. Mazurowski ,J. Y. Lo , and B. P. Harrawood, and G. D. Tourassi, "Mutual information based template matching scheme for detection of breast masses: From mammography to digital breast tomosynthesis, " Elsevier Journal of Biomedical Informatics,Vol. 44, No. 5, pp. 815-823 , San Diego , USA, October 2011.

[45] W. B. Sampaio , E. M. Diniz, A. C. Silva ,A. C. D. Paiva, and M. Gattass, "Detection of masses in mammogram images using CNN, geostatistic functions and SVM, ” Elsevier Computers in Biology and Medicine, Vol. 41, No.8, pp. 653-664, 2011.

[46] C. H. Wei , S. Y. Chen , and X. Liu, “ Mammogram retrieval on similar mass lesions, "Elsevier Computer Methods and Programs in Biomedicine, Vol. 106 , No. 3, pp. 234-248, 2012.

[47] L. Tsochatzidis, K. Zagoris, M. Savelonas, and I. Pratikakis, “ SVM - based CBIR of Breast Masses on Mammograms, ” Proc. European Conference on Artificial Intelligence (ECAI) , Vol. , No. , pp. 26-30,2014.

[48] Y. Z. Hsieh, C. M. Su , and P. C. Wang " A PSO - based rule extractor for medical diagnosis,” Elsevier Journal of Biomedical Informatics, Vol. 49 , pp. - 53- 60, June 2014.

[49] J. Dheeba, N. A. Singh, and S. T. Selvi "“ Computer-aided detection of breast cancer on mammograms: A swarm intelligence optimize wavelet neural network approach," Elsevier Journal of Biomedical Informatics, Vol. 49, pp. 45-52, February 2014

[50] K. Seetharaman , and S. Sathiamoorthy “ A unified learning framework for content based medical image retrieval using a statistical model” , Journal of King Saud University- Computer and Information Sciences, Vol. 28, No. 1, pp. 110-124, January , 2016 .

[51] S. R. Dubey , S. K. Singh , and R. K. Singh , “ Local Bit-plane decoded pattern: A Novel Feature Descriptor for Biomedical Image Retrieval,” IEEE Journal of Biomedical and Health Informatics , Vol. 20 , No. 4, pp. 1139-1147 , 2016

[52] A. K. Singh, and B. Gupta, "A Novel Approach for Breast Cancer Detection and Segmentation in Mammogram,” Proc. Eleventh International Multi Conference on Information Processing, Vol. 54 , No.2 , pp. -676-682 , 2015.

[53] P. V. Anuradha , B. R. Jose, and J. Mathew ,“ Improved Segmentation of Suspicious Regions of Masses in Mammograms by Watershed Transform,” Elsevier Procedia Computer Science, Vol. 46 , pp. 1483-1490 , 2015

[54] M. Pratiwi, Alexander, J. Harefa, and S. Nanda , "Mammograms Classification using Gray -level Co-occurrence Matrix and Radial Basis Function Neural Network, ” Proc. International Conference on Computer Science and Computational Intelligence (ICCSCI 2015), Elsevier Procedia Computer Science, Vol. 59 , No. , pp. 83-91, 2015.

[55] A. S. Setiawan , Elysia , J. Wesley, and Y. Purnama, "Mammogram Classification using Law's Texture Energy Measure and Neural Network", Proc. International Conference on Computer Science and Computational Intelligence (ICCSCI 2015) , Elsevier Procedia Computer Science , Vol. 59 , pp. 9297, 2015.

[56] H. Muller, W. Muller, D. M. Squire, S. M. Maillet, and T. Pun, " Performance evaluation in content based image retrieval : overview and proposals,"Elsevier Pattern Recognition Letters, Vol. 22, No. , pp. 593-601, 2001. 